\title{
Análisis bibliométrico de los artículos publicados en la revista Minería y Geología en el período 2000-2010
}

\author{
Arianna AGUIRRE ARAUZ \\ Especialista en Gestión Ambiental para la Ciencia, Tecnología y Medio Ambiente de Holguín, Cuba \\ Roelvis ORTIZ NUÑEZ \\ Departamento de Ciencias de la Información \\ Instituto Superior Minero Metalúrgico de Moa, Cuba. \\ rortiz@ismm.edu.cu
}

Recibido: Febrero 2013

Aceptado: Mayo 2013

\begin{abstract}
Resumen: Se realiza un estudio bibliométrico para medir el comportamiento de la producción científica de los investigadores del Instituto Superior Minero Metalúrgico de Moa, a través de los artículos publicados en la revista Minería y Geología en el período 2000-2010. Se presenta al grupo de investigadores que publican en esta revista, así como la cuantificación de los títulos de los trabajos publicados por ellos; se aplican técnicas bibliométricas para su estudio. Del análisis se obtienen resultados sobre la productividad científica de los autores, la productividad científica anual, el comportamiento de la productividad científica por departamentos docentes y la temática sobre la que más se publica. Se registran un total de 237 publicaciones en el período estudiado y resulta ser el año 2000 el más productivo de los estudiados.
\end{abstract}

Palabras clave: producción científica; Minería y Geología; Bibliometría; técnicas bibliométricas; producción temática.

\section{Bibliometric analysis of the published articles in the Mineria y Geología magazine in the period 2000-2010}

\begin{abstract}
Is carried out a bibliometric study to measure the behavior of the scientific production of the investigators of the Higher Institute Mining and Metallurgist of Moa, through the articles published in the magazine Mining and Geology in the period 2000-2010. Is presented to the group of investigators that publish in this magazine, as well as the quantification of the works titles published by them; technical bibliometrics is applied for its study. Of the analysis are obtained results about the scientific productivity of the authors, the annual scientific productivity, the behavior of the scientific productivity for educational departments and the thematic about that more is published. A total of 237 publications are register in the studied period and the year 2000 is the most productive of those studied.
\end{abstract}

Keywords: scientific production; Minería y Geología; bibliometric; Bibliometric techniques; thematic production. 


\section{INTRODUCCIÓN}

A partir de la Segunda Guerra Mundial la ciencia y la tecnología se convierten en un fenómeno masivo y complejo, en este período aparecen nuevas disciplinas y el número de científicos se incrementa, razón por la cual la publicación de trabajos científicos, materiales de eventos, patentes y otros tipos de documentos también crecen; la producción científica asciende a niveles insospechados, debido a éste fenómeno, la ciencia se desarrolla como una esfera relevante en el contexto internacional y como expresión de un área que produce conocimientos con suficientes influencias para el desarrollo tecnológico.

En el siglo XIX se produce una de las innovaciones más notables y significativas: la investigación, que unida a la enseñanza, comienza a ser apoyada por los gobiernos y se fundan Asociaciones Científicas Profesionales. Esto trajo consigo un aumento en las publicaciones de artículos y con ellas la necesidad de crear un medio para dar a conocer las investigaciones desarrolladas en el seno de la comunidad científica.

En la actualidad la actividad científica presenta un inapreciable valor que revela las contribuciones sucesivas de la comunidad de investigadores, que comunican sus progresos en diferentes instancias, lo que permite evaluar la calidad y cantidad de publicaciones que se realizan, de acuerdo con el nivel de desarrollo de cada investigador o grupos de investigadores.

Hoy se experimenta un rápido proceso del crecimiento y segmentación de la ciencia por lo que las diferentes disciplinas se expanden con rapidez y cada una de ellas necesita una rápida publicación, para de esta manera popularizar el resultado de sus investigaciones. Una de las modalidades de publicaciones sobre la que más se trabaja es la Revista Científica, cuya aparición trajo consigo la génesis de una nueva era en la comunicación, por conducir, diseminar y preservar la información científica y por representar un medio que facilita su progreso.

Existen diversos conceptos de Revista Científica, y dentro de ellos, el más general que las define como publicaciones periódicas cuyo objetivo esencial es la comunicación y transmisión del conocimiento científico y su constancia metodológica. (Guerra Pérez, 2007).

La revista científica emerge de la necesidad de divulgar el trabajo científico de los investigadores. Consecuentemente, los científicos comienzan a tener su prestigio subordinado a la reputación de la revista a la cual enviaban sus artículos; la distinción en la calidad de las revistas también comienza a ser tenida en cuenta para la evaluación de las condiciones académicas de los científicos y sobre todo, el prestigio de las revistas desempeña un papel muy significativo en la evaluación de la producción científica y de todo el sistema científico-técnico. 
Estos cambios provocan la necesidad de conocer qué se produce y cómo se produce, por lo que en los últimos años la producción científica ha sido objeto de estudio de las disciplinas métricas que se han encargado de aplicar nuevos indicadores que permiten identificar su comportamiento, tales como: autores más productivos, años de mayor producción, departamentos que más publican, entre otros, posibilitando además, medir el estado general de lo producido en determinados períodos apoyándose en la evaluación de la actividad científica.

Dentro de las instituciones más productivas en el ámbito de las ciencias se encuentran las universidades, desde donde emerge gran parte del conocimiento científico de un país, caracterizándose estas por formar profesionales en disímiles ramas del saber, a partir de las diversas investigaciones que permiten dicha formación. Por lo que se hace necesario la evaluación de la investigación, para conocer su evolución y estado actual, y su tributo al desarrollo de la ciencia en determinado campo de conocimiento.

La evaluación de la actividad científica de las universidades nos permiten conocer, ¿qué se está produciendo?, ¿cómo se está produciendo? y ¿quiénes los están produciendo? de ahí la gran importancia de realizar estudios que nos permitan analizar y conocer el comportamiento de su producción científica.

En el Instituto Superior Minero Metalúrgico de Moa, el conocimiento de la producción científica ha estado sujeta a la carencia de un estudio que refleje su comportamiento, para ello realizaremos un análisis de los artículos publicados en la Revista Minería y Geología en el periodo del 2000-2010, el cual permitirá determinar, los autores más productivos, los años de mayor producción, las publicaciones por temáticas y por departamentos docentes.

\section{OBJETIVOS}

Para dar respuesta a esta necesidad, la presente investigación propone los siguientes objetivos:

1 Realizar un estudio bibliométrico de la Revista Minería y Geología en el período 2000-2010, para determinar el comportamiento de la producción científica.

2 Cuáles son los aspectos teórico conceptuales que sustentan la producción científica en la Revista Minería y Geología del Instituto Superior Minero Metalúrgico de Moa.

3 Cuáles son los indicadores de la producción científica en la revista Minería y Geología del Instituto Superior Minero Metalúrgico de Moa.

4 Cómo se comporta la producción científica en la Revista Minería y Geología del Instituto Superior Minero Metalúrgico de Moa. 


\section{PRODUCCIÓN CIENTÍFICA}

Después de haber analizado el conjunto de conceptos dados por los siguientes autores: Cardoso Chaves (2000), Guerra Pérez, (2007), Núñez Jover (2004) y Piedra Salomón (2006) y en aras de esclarecer qué es la Producción Científica a los efectos de la presente investigación, se propone a continuación la siguiente definición:

La Producción Científica es el resultado del proceso investigativo y de la práctica laboral en cualquier campo del conocimiento científico; es la forma de inmortalizar el saber científico; es la base de la dinámica científica, como el punto de partida para la generación de nuevos conocimientos, que lleva implícito el desarrollo continuo del campo del saber. Es el criterio fundamental por el cual medir la actividad investigativa de un profesional. La producción científica se encuentra indistintamente presente en todos los soportes y canales de información.

Varios autores consideran la Producción Científica como la propia esencia de las universidades. Para Witter (1997) esta es "la forma mediante la cual una universidad o institución de investigación se hace presente a la hora de hacer ciencia, es una base para el desenvolvimiento y la superación de dependencia entre países y regiones de un mismo país; es un vehículo para la mejora de la calidad de vida de los habitantes de un país, es una forma de hacerse presente no solo hoy, sino también mañana".

Kusch (2005) expresa: lo que se desea y defiende es que" la universidad, como centro de producción sistematizada de conocimiento, canalice sus potencialidades, sus programas de naturaleza científica y cultural, procurando difundir junto a la opinión pública el saber y los progresos, los debates y las discusiones que generan las áreas de ciencias, tecnología, letras y artes. Como programas comunicacionales basados en una producción científica bien elaborada, la universidad mantendrá o recuperará su real dimensión."

La producción científica es imprescindible para conocer el desarrollo de los investigadores, de las instituciones y la expansión del conocimiento científico.

\section{MATERIAL Y MÉTODOS}

Para la investigación que se presenta, se tomó como fuente documental y de análisis los artículos de la revista Minería y Geología, publicación científica trimestral fundada el 9 de mayo de 1983 por el Ministerio de Educación Superior. Editada por el Instituto Superior Minero Metalúrgico de Moa. Su objetivo fundamental es difundir artículos inéditos de investigaciones relacionadas con las Ciencias de la Tierra y afines. En ella se publican artículos inéditos originales, artículos de revisión y réplicas de publicaciones de la propia revista, así como otras informaciones científicas de interés a los profesionales de las geociencias. 
Es una revista de alcance internacional, indexada en Bases de Datos de prestigio internacional: REDALYC, LATINDEX, ULRICH'S PERIODICALS DIRECTORY, EBSCO Fuente Académica, Actualidad Iberoamericana, ACADEMIC SEARCH COMPLETE, CAB Abstracts Database, Directory of Open Access Journals y JournalTOCs.

\subsection{CRITERIOS DE SELECCIÓN PARA EL ANÁLISIS DEL OBJETO DE ESTUDIO}

Los criterios que se tomaron en cuenta para el análisis del objeto de estudio fueron:

1. Tratamiento temático: se consideraron exclusivamente los artículos científicos de la revista Minería y Geología, objeto de investigación, que pertenecen a los investigadores del Instituto Superior Minero Metalúrgico de Moa, ya que se encontraron otros artículos de autores que no pertenecen a la institución antes mencionada.

2. Período de tiempo: se analizó un período de 11 años, comprendido entre 2000 y el 2010, con el objetivo de realizar un análisis más profundo.

3. Disponibilidad de los documentos: se consideró analizar la revista Minería y Geología, teniendo en cuenta su accesibilidad en diversos soportes y que es editada por la propia institución que forma parte del objeto de estudio además de sus características editoriales y poligráficas que las convierten en documentos de fácil manejo.

4. Relevancia del estudio: se determinó analizar a esta revista por su importancia, ya que es una revista científica académica sobre la rama geólogo-minero-metalúrgica, única de su tipo en Cuba, la misma conserva un incalculable acervo de información y conocimiento científico que la convierte en una revista muy peculiar, aunque no suficientemente valorada y validada.

5. Repercusión científica e investigativa del estudio: Se valoró la repercusión investigativa que tendría el estudio para el grupo de investigación del Instituto Superior Minero Metalúrgico que publican artículos en la revista Minería y Geología, obteniendo como resultado, un reconocimiento de sus investigaciones en forma de publicaciones. Además de dar a conocer cuáles son los investigadores que más publican y en qué temáticas lo hacen. Abre paso a nuevos estudios sobre producción científica no solo en la institución sino en el país. 


\subsection{DEFINICIÓN DE LAS VARIABLES E INDICADORES}

Se exponen las definiciones y la operacionalización de la variable; así como de sus dimensiones y sus correspondientes indicadores.

Variable teórica: Producción científica.

Definición: "forma mediante la cual una universidad o institución de investigación se hace presente a la hora de hacer ciencia, es una base para el desenvolvimiento y la superación de dependencia entre países y regiones de un mismo país; es un vehículo para la mejoría de la calidad de vida de los habitantes de un país, es una forma de hacerse presente no solo hoy, sino también mañana". (Witter, 1997).

Variable real: comportamiento de la producción de artículos científicos.

Definición: forma en que se manifiesta o refleja la producción científica universitaria en los artículos de la revista estudiada.

Para la realización de esta investigación se utilizaron indicadores de productividad científica tales como:

- Productividad científica por autores: cantidad de trabajos firmados por cada autor en el período analizado.

- Con este indicador se conoce la cantidad de contribuciones hechas por cada autor.

- Productividad científica por año de publicación: cantidad de contribuciones por año de publicación.

- Con este indicador se obtiene la cantidad de publicaciones por año.

- Productividad científica por Departamentos Docentes: cantidad de publicaciones por departamento docente.

- Con este indicador se obtendrá la cantidad de publicaciones por departamento docente.

- Productividad Temática. Indicador que mide las temáticas más representativas en la literatura localizada.

- Con este indicador se obtendrá la cantidad de artículos por temática.

\section{RESULTADOS Y DISCUSIÓN}

Este estudio se realiza tomando como base los artículos publicados en esta revista, en el periodo del 2000-2010 pero sólo se estudian 9 años ya que en el 2002 no se editó la revista.

En la investigación se analizaron las publicaciones generadas, en el período 2000-2010, donde se comprobó que en la revista publican un total de 278 investigadores, para este estudio se tomó una muestra de 237 investigadores que representa el $85,25 \%$ del total, las publicaciones que no se revisaron es por que los investigadores no pertenecen al ISMM. 
Para el estudio se analizó un total de 210 artículos publicados en la revista Minería y Geología en el periodo del 2000-2010.

\subsection{PRODUCTIVIDAD CIENTÍFICA POR AUTORES}

Se realizó el estudio a un total de 237 autores y coautores (Véase Anexo 1) en los 210 artículos analizados. Esta información nos sirvió para conocer cuáles son los investigadores más productivos en el período 2000-2010.

De acuerdo a la Ley de Lotka y a los niveles que esta propone, se realizó el listado de los autores, de manera que fue posible identificar los más productivos. Sobre la base del índice de Lotka se acostumbra a distribuir a los autores de un conjunto determinado de publicaciones en tres niveles de productividad: pequeños productores (con un solo trabajo e índice de productividad igual a 0), medianos productores (entre 2 y 9 trabajos e índice de productividad mayor que 0 y menor que 1) y grandes productores (10 o más trabajos e índice de productividad igual o mayor que 1) (Spinak, 1996).

Para la determinación de este indicador se realizó el conteo completo, donde una misma publicación firmada por autores y coautores se le asigna a cada uno de ellos, por lo que el total de las contribuciones hechas por ellos es de $237 \mathrm{y} \mathrm{el}$ total real de artículos analizados es 210 .

Tabla 1. Productividad autoral por niveles

\begin{tabular}{|c|c|c|}
\hline Nivel de productividad & Autores por nivel & Publicaciones por nivel \\
\hline $\begin{array}{c}\text { 1er nivel(10 o más } \\
\text { publicaciones) }\end{array}$ & 5 & 59 \\
\hline 2do nivel(2-9 publicaciones) & 189 & 108 \\
\hline 3er nivel(1 publicación) & 43 & 43 \\
\hline Total & $\mathbf{2 3 7}$ & $\mathbf{2 1 0}$ \\
\hline
\end{tabular}

Al observar los datos aportados por la aplicación de la Ley de Lotka y compararlos con el listado de autores, se obtiene que los grandes productores son:

Tabla 2. Autores más productivos

\begin{tabular}{|c|c|c|}
\hline Autores & \# de artículos & $\mathbf{\%}$ \\
\hline José Alberto Batista Rodríguez & 13 & 6,25 \\
\hline $\begin{array}{c}\text { Arístides Alejandro Legrá } \\
\text { Lobaina }\end{array}$ & 13 & 6,25 \\
\hline Adrián Martínez Vargas & 13 & 6,25 \\
\hline Roberto Díaz Martínez & 10 & 4,80 \\
\hline Rafael Guardado Lacaba & 10 & 4,80 \\
\hline Total & $\mathbf{5 9}$ & $\mathbf{4 8 , 0 5}$ \\
\hline
\end{tabular}

En la tabla se observan los autores, organizados según su nivel de productividad científica y dentro de ella por orden alfabético. Aparecen como mayores productores José Alberto Batista Rodríguez, Arístides Alejandro Legrá 
Lobaina y Adrián Martínez Vargas, con un total de 13 publicaciones cada uno, que representa un $18.75 \%$ del total de las publicaciones.

La gráfica 1 muestra la cantidad de artículos publicados por los autores identificados y el porciento correspondiente. En este sentido la cima está compartida por tres autores Adrián Martínez Vargas, Arístides Alejandro Legrá Lobaina y José Alberto Batista Rodríguez, con total de 13 artículos, que representan el $6,2 \%$.

Figura 1. Productividad científica por autores

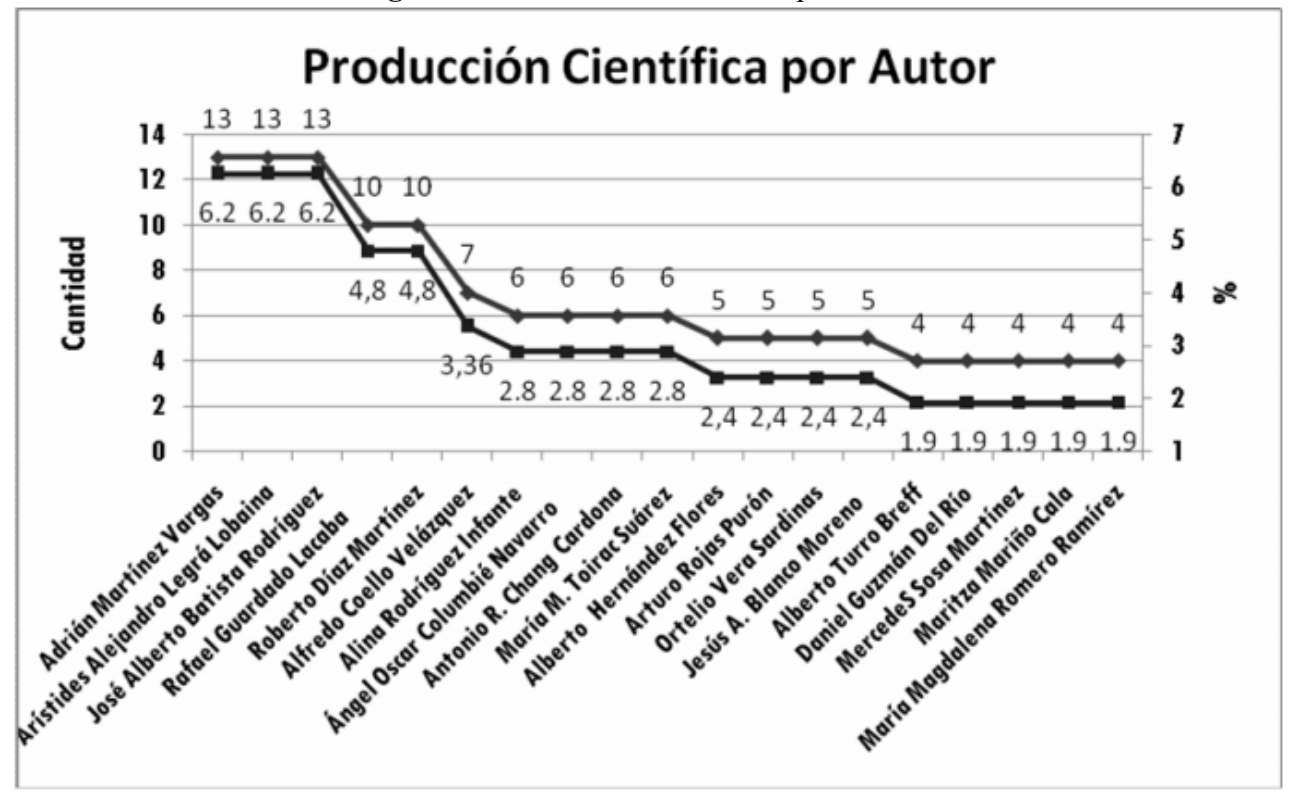

\subsection{PRODUCTIVIDAD CIENTÍFICA POR AÑO}

Por la importancia que representa para el análisis bibliométrico de la revista Minería y Geología el estudio sobre el comportamiento de las publicaciones por año en el período analizado, a continuación se muestra gráficamente la productividad científica por año, resultante del análisis bibliométrico de los artículos publicados en la revista Minería y geología, en el período 2000 - 2010. 
Figura 2. Productividad por años

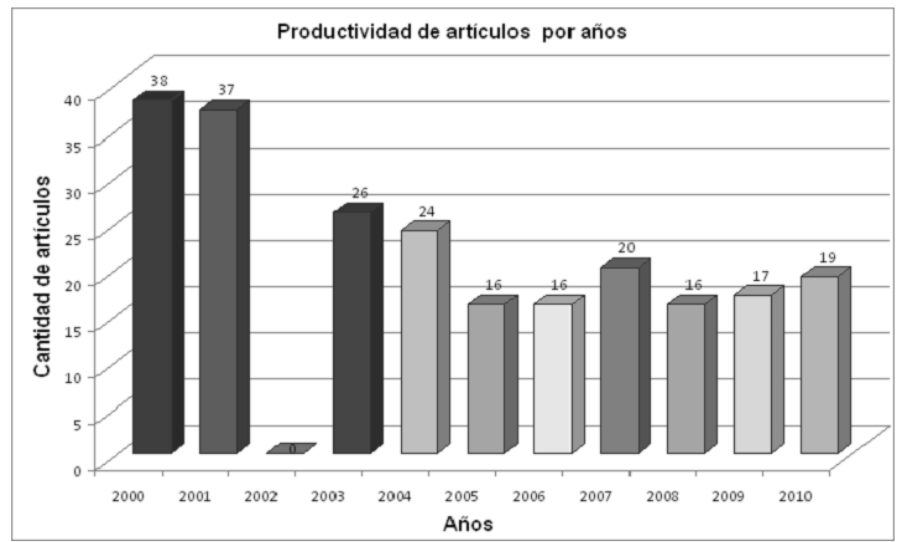

En el gráfico anterior se refleja como se ha comportado la producción científica por años en el período 2000 - 2010 resultado del análisis bibliométrico de los artículos publicados en la revista Minería y Geología en este periodo, se empleo el conteo completo de los artículos publicados por año y se obtuvo como resultado 229 artículos, pero solo se analizan 210 publicaciones que son las que pertenecen a los investigadores del Instituto Superior Minero Metalúrgico de Moa.

En el gráfico se observa como el año más productivo el 2000, con 38 publicaciones, se representa el año 2002 con un valor nulo, ya que en este año no se publicaron artículos, lo cual constituye una limitante para el estudio en cuestión y en los años del 2003-2010 ocurre un descenso en el número de publicaciones, siendo los años 2005, 2006 y 2008 los menos productivos con un total de 16 publicaciones cada uno.

\subsection{PRODUCTIVIDAD CIENTÍFICA POR DEPARTAMENTO DOCENTE}

El gráfico muestra la productividad científica por Departamentos Docentes, a los que pertenecen los autores del estudio, los investigadores del ISMM que publican en la revista Minería y Geología, por lo que no se recogen todos los departamentos.

El departamento más productivo es el departamento de Geología con un total de 74 publicaciones que representa el $35,23 \%$ del total de las publicaciones analizadas en este período y el menos productivo es el departamento de Marxismo con 5 publicaciones para un 2,38\%. 
Figura 3. Publicaciones por Departamentos Docentes

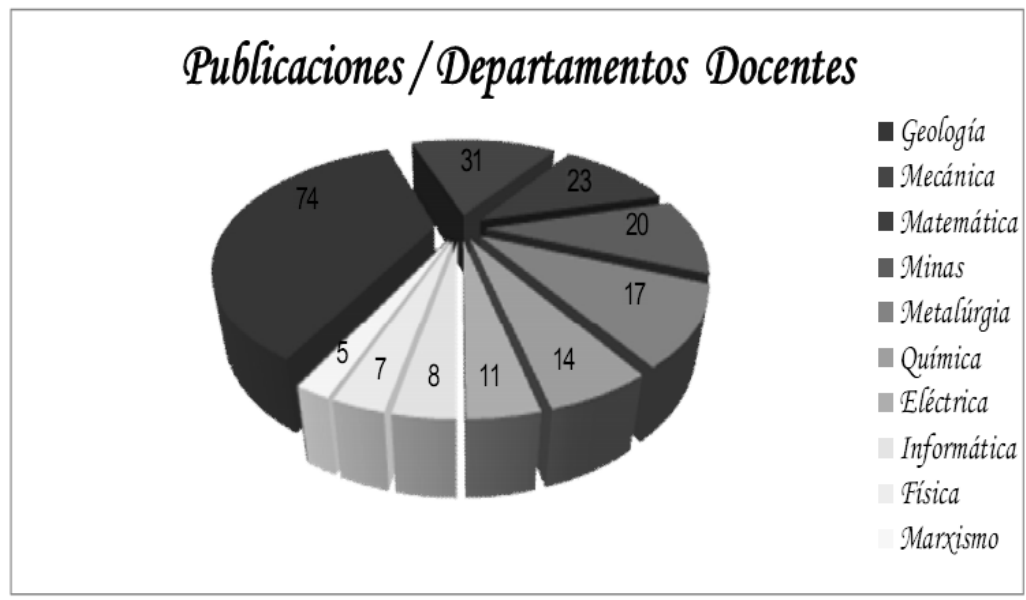

\subsection{PRODUCTIVIDAD CIENTÍFICA POR TEMÁTICAS DE INVESTIGACIÓN}

La revista Minería y Geología publica artículos científicos resultados de investigaciones de la rama geólogo-minero-metalúrgica en las temáticas:

Tabla 3. Productividad por Temáticas

\begin{tabular}{|c|c|}
\hline Temáticas & \# de publicaciones \\
\hline Georrecursos & 62 \\
\hline Preparación mecánica y beneficios & 41 \\
\hline Exploración y explotación de yacimientos & 26 \\
\hline Prospección & 24 \\
\hline Automatización y conservación energética & 23 \\
\hline Metalurgia ferrosa y no ferrosa y aleaciones & 19 \\
\hline Electromecánica & 15 \\
\hline Total & $\mathbf{2 1 0}$ \\
\hline
\end{tabular}

En la tabla anterior se muestran la producción científica por temáticas. Se destaca Georrecursos con 62 publicaciones como la temática de investigación sobre la que más se publican artículos, representando un $26,16 \%$ del total y Electromecánica con 15 artículos que representa un 7,14\% como la temática en la que menos se publica.

Llegado a este punto de la investigación es posible generalizar el comportamiento de la producción científica en 10 años (2000-2010) de publicación con los porcientos correspondientes (ver gráfico 3), donde se puede observar que el año 2000 constituye el más productivo con un total de 38 artículos publicados, lo que representa el 17,43\%, seguido muy de cerca por el 2001 con 37 artículos, que representa el 16,97\%, el año 
2002 constituye un año infructífero con 0 artículos publicados y en los años que le suceden se observa una inestabilidad en dicha producción.

Figura 4. Producción científica de la revista Minería y Geología

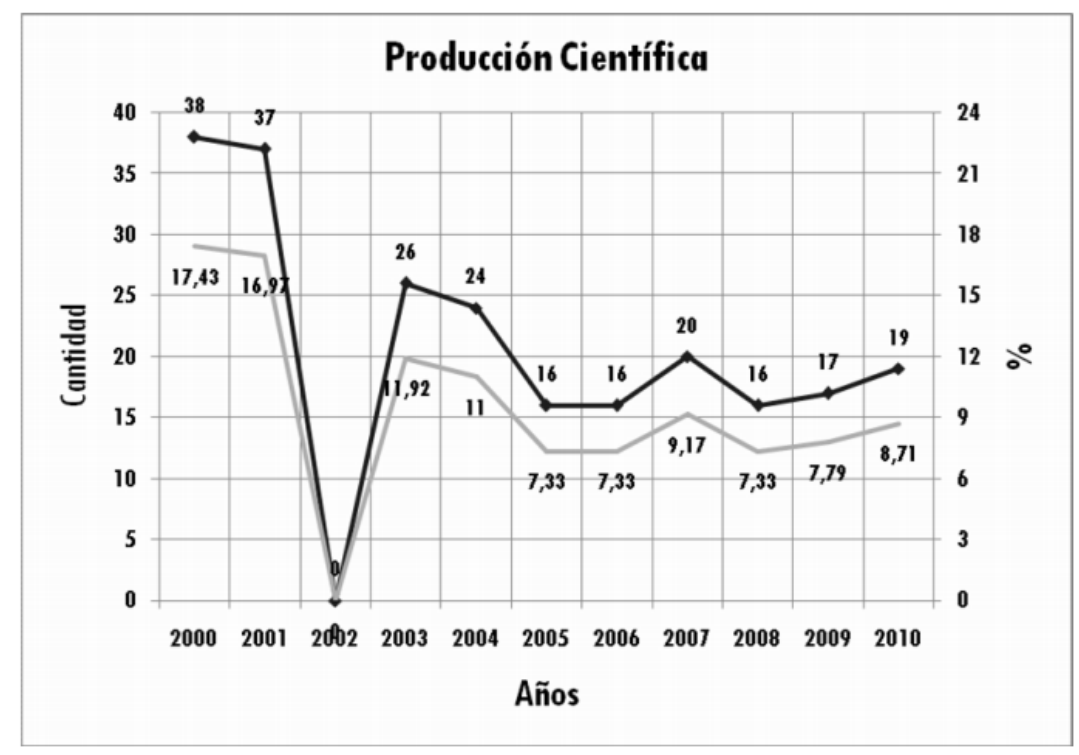

\section{CONCLUSIONES}

Se registraron un total de 237 publicaciones como parte de la producción científica de los investigadores del ISMM a través del análisis bibliométrico de la revista Minería y Geología en el período 2000-2010.

Se consideran los niveles de productividad establecidos por la ley de Lotka, se reconocen como autores más productivos a Adrián Martínez Vargas, Arístides Alejandro Legrá Lobaina y José Alberto Batista Rodríguez, con 13 publicaciones cada uno, que representa un $18.75 \%$ del total de las publicaciones sobre la base del conteo completo.

Resultó ser el 2000 el año más productivo y los menos productivos el 2005, 2006 y 2008 evidenciando un decrecimiento significativo en la producción científica.

El departamento más productivo resultó ser el de Geología con un total de 74 publicaciones que representa el 35,23\% del total de las publicaciones.

La temática sobre la que más se investiga es Georrecursos con 62 artículos publicados que representa $26,16 \%$ del total y la temática sobre la que menos se investiga es Electromecánica con 15 artículos que representa un 7,14\%. 


\section{REFERENCIAS BIBLIOGRÁFICAS}

ACUÑA, F. (2000). "La calidad de las revistas científicas y el uso del Science Citation Index". Nexos, 18(14), 11-13.

GUERRA PÉREZ, M. (2007). "Comportamiento de la productividad y la autoría en las revistas cubanas especializadas en Bibliotecología y Ciencia de la Información en el período 2000-2006". Acimed, Vol.16; No.6 [Consultado: 16 /01/2007]. Disponible en: $<\mathrm{http}: / /$ bvs.sld.cu/revistas/aci/vol16_6_07/aci071207.htm>

CAROSO CHÁVEZ, Y. (2000). "Estudio informétrico de la producción científica sobre vacunas en los temas Vibrio Cholenae, Haemophilus influenzae y Streptococcus pneumoniae para el laboratorio de antígenos sintéticos". Trabajo de Diploma. Universidad de La Habana, Facultad de Comunicación.

KROHLING KUSCH, M. A. (2005). "Produção científica em relações públicas e comunicação organizacional no Brasil: análise, tendências e perspectivas". ALAIC, [en línea]. [Consultado: 14/01/2010], 10(1). Disponible en: $<$ http://www.eca.usp.br/alaic/boletin11/kunsch.ht>

MARTÍNEZ RODRÍGUEZ, A. (2004). Estudios métricos de la información. Selección de lecturas. La Habana: Félix Varela.

NUÑEZ JOVER, J. (2004). La Ciencia y la Tecnología como procesos sociales: lo que la educación científica no debería olvidar. La Habana: Félix Varela. (26 - 36).

PHELAM, T. J. (2000). Evaluation of Scientific Productivity. The Scientist, 14 (19), 39.

PIEDRA SALOMON, Y. [et al]. (2006). Contribuciones cortas: Análisis métrico de la producción científica en comunicación social en Cuba. 14(4). Disponible en: <http://bvs.sld.cu/revista/aci/vol14-4-06/aci07406.htm\#cargo> [Consultado: 28/02/2011]

SPINAK, E. (1996). Diccionario enciclopédico de bibliometría, cienciometría e informetría, Estados Unidos: Washington, D. C.

WITTER, G. (1997). Produção científica. Campinas, SP: Editora Átomo, 1997. P.23.

\section{ANEXOS}

Anexo1: Tabla de producción científica de los autores del ISMM

\begin{tabular}{|c|c|c|}
\hline Autores & \# de artículos & \% \\
\hline Adrián Martínez Vargas & 13 & 6,25 \\
\hline Arístides Alejandro Legrá Lobaina & 13 & 6,25 \\
\hline José Alberto Batista Rodríguez & 13 & 6,25 \\
\hline Rafael Guardado Lacaba & 10 & 4,80 \\
\hline Roberto Díaz Martínez & 10 & 4,80 \\
\hline Alfredo Coello Velázquez & 7 & 3,36 \\
\hline Alina Rodríguez Infante & 6 & 2,88 \\
\hline Ángel Oscar Columbié Navarro & 6 & 2,88 \\
\hline Antonio R. Chang Cardona & 6 & 2,88 \\
\hline María M. Toirac Suárez & 6 & 2,88 \\
\hline Alberto Hernández Flores & 5 & 2,40 \\
\hline
\end{tabular}




\begin{tabular}{|c|c|c|}
\hline Autores & \# de artículos & $\%$ \\
\hline Luis Arturo Rojas Purón & 5 & 2,40 \\
\hline León Ortelio Vera Sardiñas & 5 & 2,40 \\
\hline Jesús A. Blanco Moreno & 5 & 2,40 \\
\hline Alberto Turro Breff & 4 & 1,92 \\
\hline Daniel Guzmán del Río & 4 & 1,92 \\
\hline Mercedes Sosa Martínez & 4 & 1,92 \\
\hline Maritza Mariño Cala & 4 & 1,92 \\
\hline María Magdalena Romero Ramírez & 4 & 1,92 \\
\hline Miguel Garrido Rodríguez & 3 & 1,44 \\
\hline Leduar Ramayo Cortez & 3 & 1,44 \\
\hline Rafael Pérez Barreto & 3 & 1,44 \\
\hline Tendai Njila & 3 & 1,44 \\
\hline José Nicolás Muñoz Gómez & 3 & 1,44 \\
\hline Beatriz Ramírez Serrano & 3 & 1,44 \\
\hline Alberto Velázquez del Rosario & 2 & 0,96 \\
\hline Antonio Rodríguez Vega & 2 & 0,96 \\
\hline Armando Cuesta Recio & 2 & 0,96 \\
\hline Constantino de Miguel Fernández & 2 & 0,96 \\
\hline Edelio Danguilletcourt Álvares & 2 & 0,96 \\
\hline Jorge Arce Molina & 2 & 0,96 \\
\hline Leomaris Domínguez González & 2 & 0,96 \\
\hline Marcos Medina Arce & 2 & 0,96 \\
\hline Maday Cartaya Pire & 2 & 0,96 \\
\hline Miguel Ángel Carballo Núñez & 2 & 0,96 \\
\hline Mirelis Rasúa López & 2 & 0,96 \\
\hline Secundino Marrero Ramírez & 2 & 0,96 \\
\hline Reynaldo Laborde Brawn & 1 & 0,48 \\
\hline Alexis Cabrales Rodríguez & 1 & 0,48 \\
\hline Aliuska Peña Reyna & 1 & 0,48 \\
\hline Delfina Guilarte Alpajón & 1 & 0,48 \\
\hline Eduardo Terrero Matos & 1 & 0,48 \\
\hline Félix Quintas Caballero & 1 & 0,48 \\
\hline Héctor Laurencio Alfonso & 1 & 0,48 \\
\hline Idania Aguilera Fernández & 1 & 0,48 \\
\hline Isabel Salgado Rodríguez & 1 & 0,48 \\
\hline Isnel Rodríguez González & 1 & 0,48 \\
\hline Israel Cruz Orosa & 1 & 0,48 \\
\hline José Alberto Pons Herrera & 1 & 0,48 \\
\hline José Anrotnio Fajardo Suárez & 1 & 0,48 \\
\hline José Daniel Ariosa Iznaga & 1 & 0,48 \\
\hline José Luis Sevila Rodríguez & 1 & 0,48 \\
\hline Juan Manuel Montero Peña & 1 & 0,48 \\
\hline Juan Rodríguez Gamboa & 1 & 0,48 \\
\hline Julieta Torres Lozano & 1 & 0,48 \\
\hline Julio César Llópiz Yurell & 1 & 0,48 \\
\hline Lourdes María García Pujadas & 1 & 0,48 \\
\hline Mayda Ulloa Carcassés & 1 & 0,48 \\
\hline Manuel Vega Almaguer & 1 & 0,48 \\
\hline
\end{tabular}




\begin{tabular}{|c|c|c|}
\hline Autores & \# de artículos & $\mathbf{\%}$ \\
\hline Mario Feliú Rosado & 1 & 0,48 \\
\hline Miguel A. Rodríguez Borroto & 1 & 0,48 \\
\hline Moraima Fernández Rodríguez & 1 & 0,48 \\
\hline Olga Angulo Leblanch & 1 & 0,48 \\
\hline Olga Vallejo Raposo & 1 & 0,48 \\
\hline Orlis E. Torres Breffe & 1 & 0,48 \\
\hline Rafael Charchaval Reyes & 1 & 0,48 \\
\hline Reinaldo Rojas Consuegra & 1 & 0,48 \\
\hline Roger Mis Allorca & 1 & 0,48 \\
\hline Roilbert Lambert Sánchez & 1 & 0,48 \\
\hline Tomás Fernández Columbié & 1 & 0,48 \\
\hline Virginia Costa LLanos & 1 & 0,48 \\
\hline Yaniel M. Vázquez Taset & 1 & 0,48 \\
\hline Yoneidis Hernández Pérez & 1 & 0,48 \\
\hline Yuri Almaguer Carmenates & 1 & 0,48 \\
\hline Total: 74 & $\mathbf{2 0 9}$ & $\mathbf{1 0 0 , 3 5}$ \\
\hline
\end{tabular}

